\title{
The evolving story of apolipoprotein L1 nephropathy: the end of the beginning
}

\author{
Parnaz Daneshpajouhnejad (1) ${ }^{1,2}$, Jeffrey B. Kopp (1) ${ }^{3}$, Cheryl A. Winkler $\mathbb{B}^{4}$ \\ and Avi Z. Rosenberg $\mathbb{D}^{1 凶}$
}

Abstract | Genetic coding variants in APOL1, which encodes apolipoprotein L1 (APOL1), were identified in 2010 and are relatively common among individuals of sub-Saharan African ancestry. Approximately $13 \%$ of African Americans carry two APOL1 risk alleles. These variants, termed G1 and $\mathrm{G} 2$, are a frequent cause of kidney disease - termed APOL1 nephropathy — that typically manifests as focal segmental glomerulosclerosis and the clinical syndrome of hypertension and arterionephrosclerosis. Cell culture studies suggest that APOL1 variants cause cell dysfunction through several processes, including alterations in cation channel activity, inflammasome activation, increased endoplasmic reticulum stress, activation of protein kinase $R$, mitochondrial dysfunction and disruption of APOL1 ubiquitinylation. Risk of APOL1 nephropathy is mostly confined to individuals with two APOL1 risk variants. However, only a minority of individuals with two APOL1 risk alleles develop kidney disease, suggesting the need for a 'second hit'. The best recognized factor responsible for this 'second hit' is a chronic viral infection, particularly HIV-1, resulting in interferon-mediated activation of the APOL1 promoter, although most individuals with APOL1 nephropathy do not have an obvious cofactor. Current therapies for APOL1 nephropathies are not adequate to halt progression of chronic kidney disease, and new targeted molecular therapies are in clinical trials.

'Department of Pathology, Johns Hopkins University School of Medicine, Baltimore, MD, USA.

${ }^{2}$ Department of Pathology, University of Pennsylvania Hospital, Philadelphia, PA, USA.

${ }^{3}$ Kidney Diseases Branch, NIDDK, NIH, Bethesda, $M D, U S A$.

${ }^{4}$ Basic Research Program, Frederick National Laboratory for Cancer Research, Frederick, $M D$, USA.

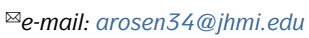
https://doi.org/10.1038/ \$41581-022-00538-3
Since its discovery in 1997 , research efforts have revealed the multifaceted nature of apolipoprotein L1 (APOL1) and, most notably, its implications for adaptation to parasitic diseases and the pathogenesis of kidney diseases. The discovery of variants in APOL1 in 2010 improved our understanding of the disproportionate prevalence of non-diabetic kidney diseases in individuals with sub-Saharan African ancestry ${ }^{1,2}$. Following the Allied victory at El Alamein in 1942, Winston Churchill said "It is not the end. It is not even the beginning of the end. But it is, perhaps, the end of the beginning ${ }^{3 "}$. Now, around a decade after the discovery of $A P O L 1$ risk variants, we have perhaps reached the end of the beginning in our task to understand the spectrum and mechanisms of APOL1 nephropathies. However, we are only now beginning the search for effective treatments.

The APOL1 risk genotypes, defined by the $\mathrm{G} 1$ and G2 risk alleles, are absent in populations without African ancestry and likely evolved to protect carriers in West Africa from African sleeping sickness. The dispersion of people from West and Central Africa to the Americas and the Caribbean islands as a consequence of the trans-Atlantic slave trade and more recent migration has led to the global distribution of APOL1 variants ${ }^{4,5}$. The alleles are therefore common among African Americans, with a combined allele frequency of approximately $34 \%{ }^{6,7}$. The association between APOL1 variants and kidney function is incompletely understood. The observation that a functional (non-pseudogenized) APOL1 gene exists in only a few African primate species and the identification of an APOL1-null individual with normal kidney function suggests that this gene is not required for normal kidney function ${ }^{8,9}$. From this perspective, the APOL1 coding variants associated with kidney disease seem to represent "gain of dysfunction" variants $^{8,10}$. Thus, natural selection in sub-Saharan African populations likely shaped this locus by balancing the beneficial effects of the variants on trypanosomal immunity with detrimental effects on kidney function ${ }^{11}$. In addition to effects on primary glomerular diseases, these genetic variants might also modify outcomes in other renal and extrarenal conditions, such as kidney allografts, coronary artery disease, cardiomyopathies, and preeclampsia ${ }^{12-15}$.

This Review summarizes insights gained from research over the past decade into the role of $A P O L 1$ variants in 


\section{Key points \\ - In contrast to other APOL family members, which are primarily intracellular, APOL1 contains a unique secretory signal peptide, resulting in its secretion into plasma. \\ - APOL1 renal risk alleles provide protection from African human trypanosomiasis but are a risk factor for progressive kidney disease in those carrying two risk alleles. \\ - APOL1 risk allele frequency is $~ 35 \%$ in the African American population in the United States, with $\sim 13 \%$ of individuals having two risk alleles; the highest allele frequencies are found in West African populations and their descendants. \\ - Cell and mouse models implicate endolysosomal and mitochondrial dysfunction, altered ion channel activity, altered autophagy, and activation of protein kinase $\mathrm{R}$ in the pathogenesis of APOL1-associated kidney disease; however, the relevance of these injury pathways to human disease has not been resolved. \\ - APOL1 kidney disease tends to be progressive, and current standard therapies are generally ineffective; targeted therapeutic strategies hold the most promise.}

kidney disease and provides context for findings that are expected to emerge in the coming years. We describe principal aspects of the APOL1 knowledge base, in particular focusing on findings most relevant to nephrology with an emphasis on our understanding of the genetics, protein structure and biological functions of $A P O L 1$ variants and their associations with cardiorenal disease.

\section{The APOL1 protein}

In 1997, researchers seeking to identify the protein components of ApoA1-containing lipoproteins - which are present in high-density lipoprotein (HDL) particles — isolated a novel protein, which was termed $\mathrm{ApoL}^{16}$. Four years later, they described three additional APOL family members, ApoL I-IV (now termed APOL 1-4). The APOL family expanded further in 2001, when two groups cloned the APOL gene cluster on chromosome 22, which also encodes APOL5 and APOL6 $\left(\mathrm{REFS}^{17,18}\right)$. Although some researchers continue to use "ApoL", consistent with protein terminology from the lipoprotein field, in this Review we use the HUGO Gene Nomenclature Committee-recommended nomenclature and style for human proteins (APOL) and genes $(A P O L)^{17}$.

$A P O L 1$ RNA is expressed in many tissues ${ }^{18}$. APOL1 is unique among the $A P O L$ genes in encoding a secretory signal peptide, resulting in secretion of APOL1 into plasma (FIG. 1a). However, APOL1 is present in humans and several Old World primates, but is otherwise absent in other mammals, indicating that APOL1 likely arose by tandem gene duplication during primate evolution around 30 million years ago ${ }^{19}$.

In 2009, studies of APOL family members showed that APOL1-6 are present in humans and that rapid evolution among simian primates occurred in domains involved in host-parasite interactions. In APOL1 for example, sequences with evidence of rapid evolution are present in or adjacent to the domain of APOL1, which interacts with the serum resistance antigen (SRA) in trypanosomes, likely reflecting a prolonged period of host-parasite interactions ${ }^{20}$.

\section{Evolution of APOL1 variants}

The high burden of CKD and kidney failure - in particular, the strikingly high frequency of focal segmental glomerulosclerosis (FSGS) and HIV-associated nephropathy (HIVAN) - among African Americans suggested that particular risk variants were likely responsible and that such variants might be enriched on Africaninherited haplotypes. In 2008, two studies employed an admixture-mapping strategy to identify chromosomal segments of African origin enriched in patients with biopsy-confirmed FSGS and non-diabetic kidney failure. One group identified a region on chromosome 22, centred on MYH9, which was strongly associated with FSGS and HIVAN; this finding was replicated in patients with hypertensive nephrosclerosis and non-diabetic kidney failure, but not in patients with diabetes-associated kidney failure ${ }^{21}$. Another group identified the same African ancestry linkage region on chromosome 22 with non-diabetic kidney failure but similarly, not in patients with diabetes-associated kidney failure ${ }^{22}$. These findings led to the discovery 2 years later of African-specific variants in the APOL1 gene, which is adjacent to MYH9. The two studies identified three APOL1 variants in close proximity in the C-terminal SRA-binding domain of APOL1 (FIG. 1a) as being primarily responsible for the association with kidney disease ${ }^{1}$. The two variant haplotypes were termed G1 and G2, with G1 (rs73885319 and rs60910145) comprising two non-synonymous coding variants (Ser342Gly, Ile384Met), and G2 (rs71785313) consisting of a six-base-pair deletion resulting in two amino acid deletions: del Asn388 and del Tyr389 ${ }^{1}$. In West Africans and their recent descendants, the two alleles (G1 and G2) form two common variant haplotypes and one infrequent haplotype (FIG. 1 b) ${ }^{13}$. The ancestral haplotype is termed G0 and does not carry G1 or $\mathrm{G} 2$ variants $^{1,13}$. In the absence of G0, the risk variants showed a strong association with FSGS, non-diabetic kidney failure and HIVAN, leading to the conclusion that they contribute to the overall higher risk of these kidney disorders in African Americans ${ }^{1,2,13}$ (FIG. 1 C).

\section{Loss of function or gain of dysfunction}

With the exception of one South African study, which showed a dominant association of G1 with HIVAN ${ }^{23}$, overwhelming evidence from case-control and cohort studies suggests that the APOL1 risk variants follow a recessive model of inheritance. Most recessive alleles are associated with a loss of gene function; however, some evidence suggests that APOL1 variants might counter this paradigm. Although at least one in vitro study did not observe toxicity following the expression of APOL1 risk variants in kidney cells, supporting a loss-of-function mechanism ${ }^{24}$, most studies suggest that APOL1 risk alleles follow a gain-of-dysfunction mechanism ${ }^{8,9}$. One model suggests that multimerization of APOL1 enables a recessive mode of inheritance with gain-of-dysfunction effects for G1 and G2 and suppression of toxicity by G0 (REF. ${ }^{25}$ ). Another study ${ }^{26}$ reported that G0 rescues $\mathrm{G} 1$ and $\mathrm{G} 2$ toxicity by transporting the variant proteins from the endoplasmic reticulum to the cytoplasm on lipid droplets; this model suggests that G0 may act as a dominant suppressor of toxicity by oligomerizing with the variant proteins and serving as a chaperone. Others have found that G1 and G2, but not G0, form multimers in the mitochondrial matrix and induce cell death by activating the mitochondrial permeability transition pore ${ }^{27}$. More recently, researchers have 
G1

Ser342Gly Ile384Met
G2

delAsn388; delTyr389

\begin{tabular}{l|l|l|l|l|l|l|}
\hline $\mathrm{N}$ terminus & \begin{tabular}{|l|l|l|l} 
Signal \\
peptide
\end{tabular} & $\begin{array}{l}\text { Pore-forming } \\
\text { domain }\end{array}$ & $\begin{array}{l}\text { BH3 } \\
\text { domain }\end{array}$ & $\begin{array}{l}\text { Membrane- } \\
\text { addressing domain }\end{array}$ & $\begin{array}{l}\text { Serum-resistance-associated } \\
\text { protein-binding domain }\end{array}$ \\
\cline { 2 - 4 }
\end{tabular}

G0 APVSFFLVLDVVYLVYESKHLHEGEKSETAEELKKVAQELEEKLNILNNNYKILQADQEL G1 ${ }^{\mathrm{GM}}$ APVGFFLVLDVVYLVYESKHLHEGEKSETAEELKKVAQELEEKLNMLNNNYKILQADQEL $\mathrm{G}^{\mathrm{Gl}}$ APVGFFLVLVVYLVYESKHLHEGEKSETAEELKKVAQELEEKLNILNNNYKILQADQEL G2 APVSFFLVLDVVYLVYESKHLHEGEKSETAEELKKVAQELEEKLNILNN--KILQADQEL

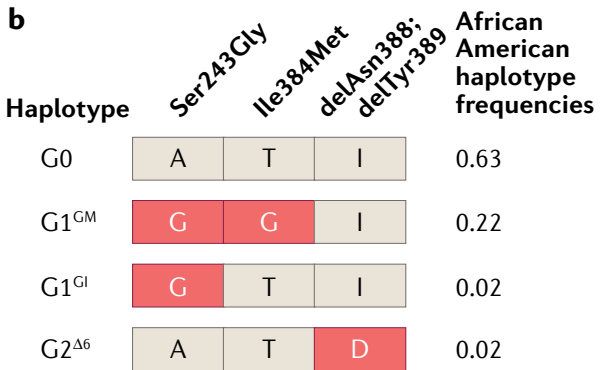

\begin{tabular}{l|l|l|l|}
\multicolumn{1}{l}{ c } & \multicolumn{1}{c}{ T.b. rhodesiense } & \multicolumn{1}{c}{ T.b. gambiense } & \multicolumn{1}{c}{ CKD } \\
\cline { 2 - 4 } G0/G0 & Susceptible to acute HAT & Susceptible to chronic HAT & Not at greater risk of CKD \\
\cline { 2 - 4 } G0/G1 & Susceptible to acute HAT & Latent asymptomatic infection & Not at greater risk of CKD \\
\cline { 2 - 4 } G1/G1 & Susceptible to acute HAT & Latent asymptomatic infection & Increased risk of CKD \\
\cline { 2 - 4 } G0/G2 & Protected against acute HAT & Susceptible to chronic HAT & Not at greater risk of CKD \\
\cline { 2 - 4 } G2/G2 & Protected against acute HAT & Susceptible to chronic HAT & Increased risk of CKD \\
\cline { 2 - 4 } G1/G2 & Protected against acute HAT & Latent asymptomatic infection & Increased risk of CKD \\
& & &
\end{tabular}

Fig. 1 | APOL1 domains and variants. a | The APOL1 protein has four functional domains and a signal peptide, which is required for secretion of liver-produced APOL1 into plasma. Forms of APOL1 that lack the signal peptide owing to alternative splicing are retained as intracellular proteins. The $\mathrm{G} 1$ and $\mathrm{G} 2$ variant isoforms are the primary drivers of APOL1-mediated chronic kidney disease (CKD). The presence of two missense mutations (Ser342Gly and lle384Met) in the nucleotide sequence encoding the serum resistance-associated protein-binding domain of APOL1 generates the G1 variant, whereas a 6 base-pair deletion that results in the loss of two amino acids (delAsn388 and delTry389) generates the G2 variant. b | The three kidney risk variants form only four observed haplotypes. Owing to the close physical proximity of the three disease-associated alleles, recombination events have not been observed and therefore the G1 and G2 alleles are mutually exclusive and do not occur together on the same chromosome. The G1 and G2 haplotypes are unique to individuals with sub-Saharan ancestry whereas the ancestral G0 haplotype is found in all global populations. Haplotype frequencies are shown for a healthy African American population?. c $\mid$ Susceptibility and resistance (indicated by red and blue shading, respectively) to acute human African trypanosomiasis (HAT) caused by Trypansoma brucei rhodesiense and chronic HAT caused by T.b. gambiense, and risk of APOL1-associated CKD, vary as a function of APOL1 haplotype. Heterozygous or homozygous carriers of the $\mathrm{G} 0$ allele are not at increased risk of kidney disease. Carriers of 1 or 2 copies of the $\mathrm{G} 1$ allele are susceptible to infection by T.b. gambiense but are less likely to develop symptoms of HAT; the mechanism of this protective association is unknown. The $\mathrm{G} 2$ variant protein efficiently lyses T.b. rhodesiense in vitro, thereby limiting infection in G2 carriers. People with G1/G1, G2/G2 and G1/G2 genotypes are at an increased risk of CKD. In certain uncommon settings (for example, in individuals with untreated $\mathrm{HIV}$ infection), $\mathrm{G} 1 / \mathrm{G} 0$ individuals may also be at an increased risk of CKD. proposed a model in which risk alleles are associated with enhanced cation permease activity compared with the reference genotype ${ }^{28}$.

Of note, a comparison of naturally occurring G1 and G2 haplotypes with the artificial haplotypes used in experimental models showed the existence of amino acid changes in other domains of the naturally occurring G1 and G2 haplotypes that were not present in the commonly used artificial, reference haplotype ${ }^{29}$. These naturally occurring, linked variants affect the properties of the expressed protein, and modify the degree of risk allele-mediated toxicity. These findings support the gain-of-toxicity hypothesis and further explain why some studies do not observe enhanced toxicity with the risk variants ${ }^{29}$

A 2020 study found that APOL1 risk alleles have dose-dependent toxic effects in human embryonic kidney HEK293 cells, resulting in loss of cell viability, cell swelling and dysregulation of energy metabolism ${ }^{30}$. These effects were not attenuated by co-expression of G0, supporting the notion that the APOL1 risk alleles acquire toxicity in a gain-of-function manner.

\section{Geographic distribution of APOL1 alleles}

The APOL $1 \mathrm{G} 1$ and $\mathrm{G} 2$ variants likely arose in West Africa after the out-of-Africa migrations 60,000 years ago ${ }^{1,31}$ and are thus found only in individuals with sub-Saharan
African ancestry. Regionally, the G1 and G2 alleles are most prevalent in West Africa, with a combined allele prevalence of over $40 \%$ among major ethnic groups in Ghana and Nigeria ${ }^{13,32-34}$. However, the distribution of allele frequencies is complex and varies widely among African ethnic populations, even within the same geographical region or country, likely because of historical endogamy within ethnic groups and migratory patterns (FIG. 2).

Trypanosoma brucei (T.b.) gambiense and T.b. rhodesiense are intracellular parasites that cause African human trypanosomiasis (African sleeping sickness). T. b. rhodesiense is distributed in eastern and southern Africa and causes the acute form of the disease, accounting for $2 \%$ of all trypanosomiasis cases. The chronic form of sleeping sickness, which is caused by T. b. gambiense, is found in west and central Africa and accounts for $98 \%$ of cases $^{35,36}$ (FIG. 2). Trypanosoma brucei brucei is a subspecies that is physically indistinguishable from the other two subspecies; it infects many vertebrates, including domestic cattle and horses $^{37}$, but not humans. This host selectivity is likely the result of co-evolution, with primates and trypanosomes each developing measures and countermeasures to ensure survival. It has been known for almost a century that some trypanosomes cannot infect humans as they are lysed by a component of normal human serum, now understood to be associated with a subclass of human $\mathrm{HDL}^{38}$. 


\section{REVIEWS}

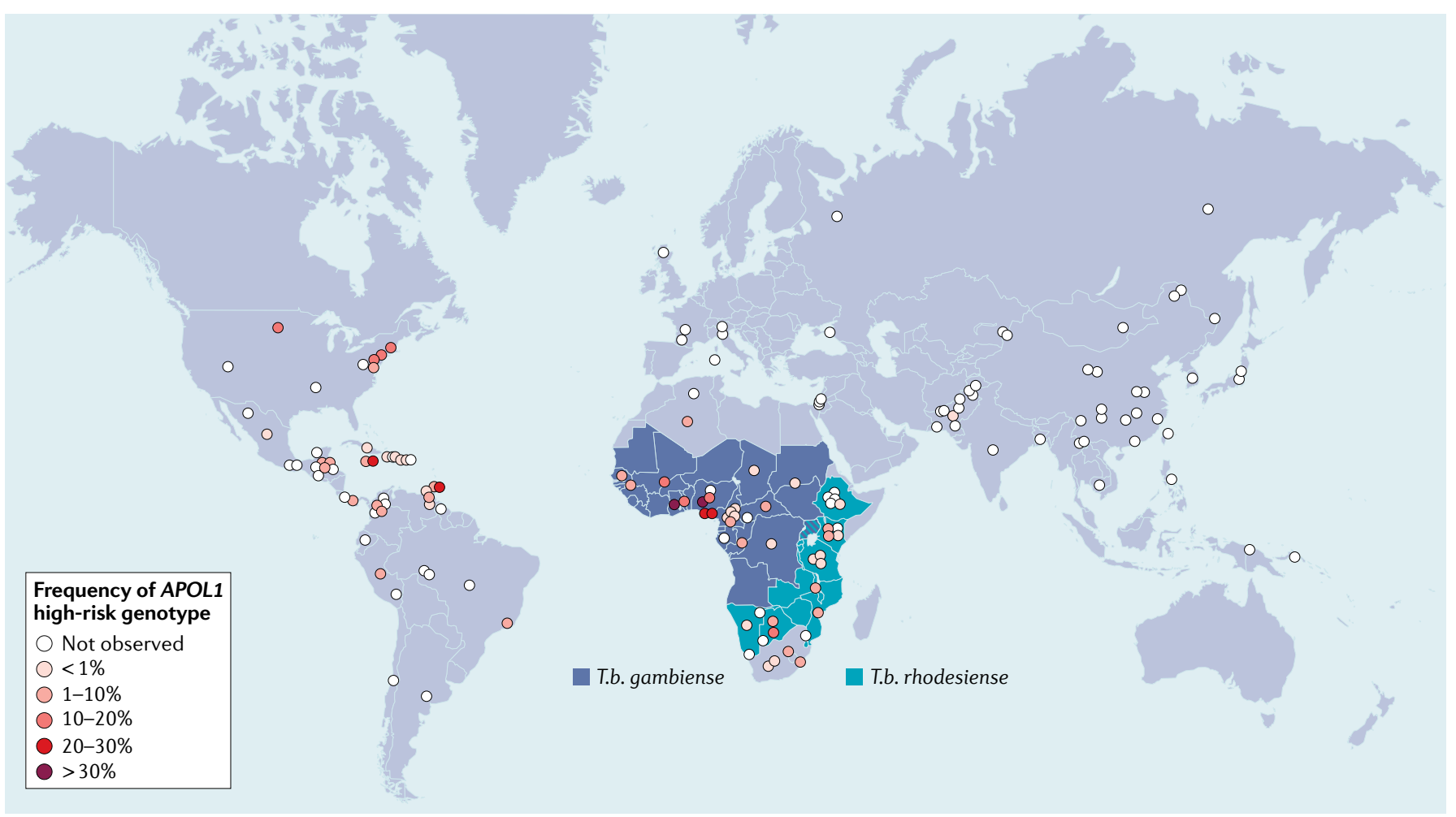

Fig. 2 | Global distribution of APOL1 high-risk genotypes and endemic areas of Trypanosoma brucei gambiense and Trypansoma brucei rhodesiense. The highest prevalence of APOL1 high-risk genotypes, defined as the presence of two risk alleles (i.e. G1/G1, G1/G2 or G2/G2), is in West Africa, but carriage of high-risk genotypes is found throughout sub-Saharan Africa and among those with African ancestry in the Americas and elsewhere. Allele frequency data are derived from Nadkarni et al. ${ }^{4}$ and Limou et al. ${ }^{7}$ ) and unpublished data (C.A. Winkler, unpublished work).

In 2003, researchers made the seminal observation that APOL1 contributes to trypanosome lysis via the actions of trypanosome lytic factors ${ }^{39}$. They also identified a T. $b$. rhodesiense gene that encodes a lysosomal SRA, which interacts with the C-terminal domain of APOL1 (FIG. 1a) and confers resistance to T. b. rhodesiense lysis by APOL1 in vitro ${ }^{39}$. Subsequent studies demonstrated that maximal anti-trypanosomal activity requires the assembly of APOL1 with other components ${ }^{40}$ present within the HDL particle and that this assembly is required to protect humans from T.b. brucei and T.b. evansi infection $^{40,41}$. The G1 missense variants and the G2 deletion occur within the SRA protein binding domain and promote APOL1 trypanolytic activity in vitro by reducing or preventing SRA binding, respectively, by T.b. rhodesiens $^{1,31}$. However, neither variant restores trypanolytic activity against T.b.gambiense ${ }^{1}$.

The extent of natural protection against human African trypanosomiasis (HAT) provided by the variant proteins are not fully understood. Although trypanosomal lytic activity has been attributed to the APOL1 G2 variant protein and, to a lesser degree, the G1 variant protein, using in vitro assays ${ }^{1}$, and delayed parasitaemia has been demonstrated in transgenic mice expressing the $A P O L 1 \mathrm{G} 1$ variant $^{31}$, the effects of these variants on natural infection and development of acute and chronic HAT caused by T.b. rhodesiense and T.b. gambiense, respectively, is not fully understood. Findings from case-control studies from HAT endemic regions of
sub-Saharan Africa indicate that protection conveyed by the G1 and G2 variants is complex (FIG. 1c). In one case-control study, the $\mathrm{G} 2$ variant prevented infection by T.b. rhodesiense, endemic to East Africa, consistent with in vitro findings. However, whereas G2 was associated with the development of symptomatic HAT in those infected by T.b. gambiense, endemic to West and Central Africa ${ }^{42}$, the G1 variant was associated with asymptomatic T.b. gambiense parasitaemia, but notably did not affect the risk of infection by either T.b. rhodesiense or T.b. gambiense ${ }^{42}$. These differential effects were confirmed by a second study, which found that the G2 variant was associated with a higher risk of developing T.b. gambiense-associated HAT, whereas the G1 variant was associated with lower risk ${ }^{43}$ (FIG. 1 C). By contrast, a 2018 study observed no significant associations between the presence of APOL1 risk alleles and HAT in two Ugandan populations, which were exposed to both trypanosomal species ${ }^{44}$. These studies suggest that the effects on trypanosome infection and HAT severity may differ regionally owing to local adaptations by the host and pathogen. The discordance between the geographic distribution of allele frequencies and trypanosomal sensitivity also raises the possibility of an as yet unidentified source of selective pressure acting on APOL1.

Thus, a complex interplay exists between APOL1 evolution and trypanosomal infections, and associated selective pressures across sub-Saharan Africa are likely the primary driver underlying the emergence of $A P O L 1$ 
risk alleles and the associated end organ toxicities observed in clinical practice today.

\section{APOL1 and kidney disease}

Following the discovery of the APOL1 variants, a number of studies reported strong associations between the $A P O L 1$ risk variants and kidney disease, particularly FSGS and HIVAN (odds ratios of 17 and 29, respectively, in the United States (and 89 for HIVAN in South Africa)), but also with sickle cell nephropathy and particular immune-mediated kidney diseases, including lupus nephritis ${ }^{13,23,45-48}$. In patients with certain forms of CKD, the presence of two APOL1 risk alleles was associated with more rapid disease progression and an increased risk of kidney failure ${ }^{49}$. Other studies showed that although few otherwise healthy, young-to-middle-aged adults with two APOL1 risk alleles, normal estimated glomerular filtration rate (eGFR) and no or low-grade proteinuria develop kidney disease during their lifetimes, some of these individuals do develop incident kidney disease $e^{9,50,51}$. These findings suggested that a "second hit" is required to initiate kidney injury in individuals with APOL1 risk genotypes ${ }^{52}$. Factors considered as second hits included genetic variants beyond APOL1, systemic factors and environmental factors ${ }^{45,53,54}$. The most established systemic factors are viral infections (for example, HIV) and, more generally, systemic elevations in interferon levels. It is now apparent that APOL1 is expressed in an interferon-dependent manner ${ }^{55}$.

In the past couple of years, $A P O L 1$ risk allele status has been observed to modify the risk of kidney injury associated with SARS-CoV-2 infection. Cases of collapsing glomerulopathy have been reported in individuals with $A P O L 1$ risk variants with COVID-19 and hyper-inflammatory features, potentially reflecting a high-interferon state ${ }^{56,57}$. Of note, however, one case report described a patient with COVID-19-related collapsing glomerulopathy in the setting of one $A P O L 1$ risk allele $^{58}$, suggesting a potential dominant effect or the existence of another unknown second hit.

Studies in the past few years have demonstrated that kidney transplant recipients with APOL1 high-risk genotype donor kidneys have lower eGFR at follow-up and poorer allograft survival ${ }^{15,59-61}$. However, whether the presence of two APOL1 risk alleles in the transplant recipient affects the outcome of the transplanted kidney is unclear, with studies showing conflicting results ${ }^{62,63}$. Furthermore, kidney donors with two APOL1 risk alleles have an increased risk of reduced kidney function following kidney donation, which has the potential to progress to kidney failure, suggesting that counselling should be considered to inform potential kidney donors with $A P O L 1$ risk variants about the associated risks ${ }^{61}$. The most compelling case for genetic testing of APOL1 in the clinical setting at present is therefore in kidney transplantation, particularly for potential living donors ${ }^{64,65}$. An ongoing NIH-funded study - the APOL1 Long-term Kidney Transplantation Outcomes study (APOLLO) - is aimed at prospectively determining outcomes in living kidney donors with African ancestry and in recipients of living and deceased donor kidneys from individuals with African ancestry, in order to better define the effect of $A P O L 1$ risk alleles on transplantation outcomes, and will provide data with which to guide patients and clinicians in judging the risk of transplantation for living donors and recipients ${ }^{66,67}$.

APOL1 risk status has also been associated with kidney disease in children, most strongly in the setting of HIV infection ${ }^{68,69}$. Reports from two large paediatric cohorts - the Chronic Kidney Disease in Children (CKiD) and the Nephrotic Syndrome Study Network (NEPTUNE) study - show that children with a high-risk genotype have a higher prevalence of FSGS than those with a low-risk genotype and that the disease often follows an aggressive course ${ }^{70}$.

To date, no significant correlation has been identified between APOL1 plasma levels and kidney function, with the possible exception of kidney transplant, as discussed below ${ }^{71}$. Moreover, studies in mouse models of APOL1-associated kidney disease have shown that expression of APOL1 risk variants in podocytes is associated with functional (albuminuria and azotaemia), histomorphological (podocyte foot-process effacement and glomerulosclerosis), and characteristic molecular (gene-expression) changes ${ }^{72}$. Together with the kidney transplant experience, these findings suggest that kidney-expressed APOL1 is the primary driver of kidney injury rather than circulating APOL1 in plasma ${ }^{15,61}$.

APOL1 localization in the kidneys. In 2011, immunohistochemistry studies demonstrated localization of APOL1 to podocytes, arteriolar endothelium and proximal tubular epithelium in kidney tissue from healthy individuals and from patients with FSGS or HIVAN ${ }^{73}$. Interestingly, and despite the preservation of podocyte markers, fewer podocytes in glomeruli from patients with FSGS and HIVAN expressed APOL1 compared with podocytes in normal kidneys. On the other hand, increased APOL1 staining was observed in the vasculature of diseased kidneys, with expression located in a subset of $\alpha$-smooth muscle actin-positive cells, the media of medium-sized arteries and arterioles. The expression patterns were similar in both HIVAN and FSGS cases, leading the researchers to propose that the robust expression of APOL1 in specific kidney cells is a marker of de novo APOL1 synthesis in kidney tissue and not a consequence of kidney deposition of plasma APOL1.

Subsequent evaluation of this hypothesis by other researchers led to the conclusion that APOL1 in kidney cells is derived from both cellular synthesis and uptake from plasma or the glomerular filtrate. Using indirect immunofluorescence microscopy of kidney tissue from healthy individuals, these researchers noted more APOL1 protein in podocytes and less protein in tubular epithelial cells. Fluorescence in situ hybridization studies revealed expression of APOL1 mRNA in podocytes, endothelial cells and proximal tubules, confirming the endogenous production of APOL1 in a restricted set of kidney cells. These results are consistent with studies of kidney-derived cell lines, in which both APOL1 mRNA and protein were detected in proximal tubular epithelial cells and glomerular endothelial cells, with lower expression in podocytes. Moreover, these studies 
demonstrated uptake of APOL1 protein by cultured podocytes, but not by cultured glomerular endothelial cells and proximal tubular cells ${ }^{74}$. Studies of transgenic zebrafish models also showed co-localization of APOL1 with podocyte markers and to a lesser extent, with endothelial cells. In podocytes, APOL1 expression was more marked in the podocyte body than in the foot processes ${ }^{75}$. Together, these data demonstrate that APOL1 production occurs in the kidney and may drive local injury.

APOL1 levels in plasma. Despite the importance of kidney-expressed APOL1 for kidney injury, the majority of APOL1 is produced in the liver and circulates in plasma. Several groups have assessed the implications of circulating APOL1 and showed that levels do not correlate with kidney disease risk, underscoring the essential role of kidney-expressed APOL1 in kidney disease ${ }^{71,76,77}$. A more recent study proposed that the circulating APOL1 variants have an immunomodulatory role, associated with $\mathrm{T}$ cell-mediated rejection and death-censored allograft loss ${ }^{63}$. Further studies are required to establish the role of circulatory APOL1 levels. Renal transplant studies provide a unique setting to distinguish between the effects of systemic APOL1 expression and kidney-limited APOL1 expression.

Models of APOL1-associated kidney disease. The $A P O L 1$ gene is absent from all experimental animals used to model human disorders and thus the development of animal models of APOL1-associated disorders has required transgenic approaches. Several groups have developed transgenic mouse models of APOL1 nephropathies. Mice with podocyte-specific expression of the APOL1 G2 variants under a nephrin promoter did not demonstrate overt manifestations of kidney disease but had a lower podocyte density than APOL1 G0-transgenic mice at about 6 months of age. However, APOL1 G2 transgenic mice, and to a lesser extent, APOL1 G0 transgenic mice, showed a pregnancy-related preeclampsia-like phenotype ${ }^{78}$. Transgenic mouse models with podocyte-specific and tubule-specific expression of APOL1 G0, G1 or G2 isoforms have also been generated using a tetracycline inducible enhanced green fluorescent protein (EGFP)-expressing plasmid vector. Mice with glomerular expression of either APOL1 risk allele, but not the common variant, developed proteinuria and glomerulosclerosis, with the extent of glomerulosclerosis correlating with the level of APOL1 expression ${ }^{72}$. By contrast, mice with tubule-specific expression of APOL1 did not demonstrate kidney abnormalities, underscoring the importance of glomerular, and particularly podocyte, APOL1 expression ${ }^{72}$. Transgenic mice with doxycycline-induced expression of APOL1 in podocytes also exhibited proteinuria and FSGS lesions ${ }^{79}$.

Other approaches to studying APOL1 kidney disease have involved the hydrodynamic delivery of human APOL1 mRNA to mice. Using this technique, mice receiving APOL1 risk variants showed more liver and kidney injury than those with the $A P O L 1 \mathrm{G} 0$ variant $^{31,41,80}$.

Other model organisms have also been used. The $z A P O L 1$ gene in zebrafish is homologous to the human

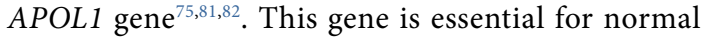
glomerular function in zebrafish, as loss of function is associated with derangement of glomerular architecture. In zebrafish with deletion of zAPOL1, expression of the human $A P O L 1 \mathrm{G} 0$ variant leads to improvement in glomerular function, whereas expression of the G1 and G2 variants do not ${ }^{82,83}$. Moreover, transgenic expression of the $\mathrm{G} 1$ and $\mathrm{G} 2$ variants result in subtle alterations in renal structure that are apparent only on electron microscopy; these alterations are not present in APOL1-G0 transgenic zebrafish. Similar findings have been reported in studies of Drosophila nephrocytes, which show functional and structural similarities to podocytes. In these cells, the expression of two APOL1 G1 and G2 variants causes defects in intra-organelle acidification, leading to cellular hypertrophy and subsequent death ${ }^{84,85}$.

\section{APOL1 disease associations beyond kidney}

APOL1 risk variants may be associated with diseases other than kidney diseases ${ }^{86}$, with some evidence supporting an association with cardiovascular diseases ${ }^{87}$, including atherosclerosis ${ }^{88,89}$, hypertension ${ }^{90,91}$, acute coronary syndrome and myocardial infarction ${ }^{88}$, stroke $^{92}$ and heart failure ${ }^{93,94}$. However, data regarding these associations are inconsistent. Although some studies have reported higher cardiovascular mortality among patients with $A P O L 1$ risk variants ${ }^{95}$, other studies, including a meta-analysis of 21,305 Black individuals from eight cohorts, do not support a strong correlation between $A P O L 1$ risk variants and cardiovascular diseases ${ }^{51,93,96-98}$.

Associations may also exist between APOL1 risk variants and preeclampsia - a pregnancy complication that is more common in populations of sub-Saharan African descent than in other populations ${ }^{99}$. A 2018 study reported that although maternal APOL1 risk genotype was not associated with preeclampsia, mothers of fetuses with two APOL1 risk alleles had a higher risk of preeclampsia than mothers of fetuses with low-risk alleles $^{14}$, with an odds ratio of 1.8. This finding was confirmed and extended in a second study, which also found that APOL1 allelic mismatch between fetus and mother was associated with nearly three-fold increased odds of preeclampsia ${ }^{100}$. However, and as described earlier, a study of APOL1 transgenic mice showed an eclampsia-like phenotype to be associated with the G2 risk allele and also unexpectedly with the G0 genotype; the effect of the G1 risk allele was not studied ${ }^{78}$. The occurrence of a preeclampsia phenotype in dams lacking an orthologous APOL1 gene may be due to expression of the fetal APOL1 transgene in the placenta, resulting in a fetal-maternal incompatibility, or to the general toxicity of high levels of APOL1 protein, irrespective of genotype.

Other studies have also demonstrated an association of APOL1 genotype with sepsis in Black adults ${ }^{101,102}$. The mechanism for this effect remains obscure and warrants further attention.

\section{Mechanisms of cell injury}

Various mechanisms of APOL1-mediated cell toxicity have been proposed that might underlie the association between the APOL1 risk variants and kidney disease. Several of these are outlined below (FIG. 3; TABLE 1). 

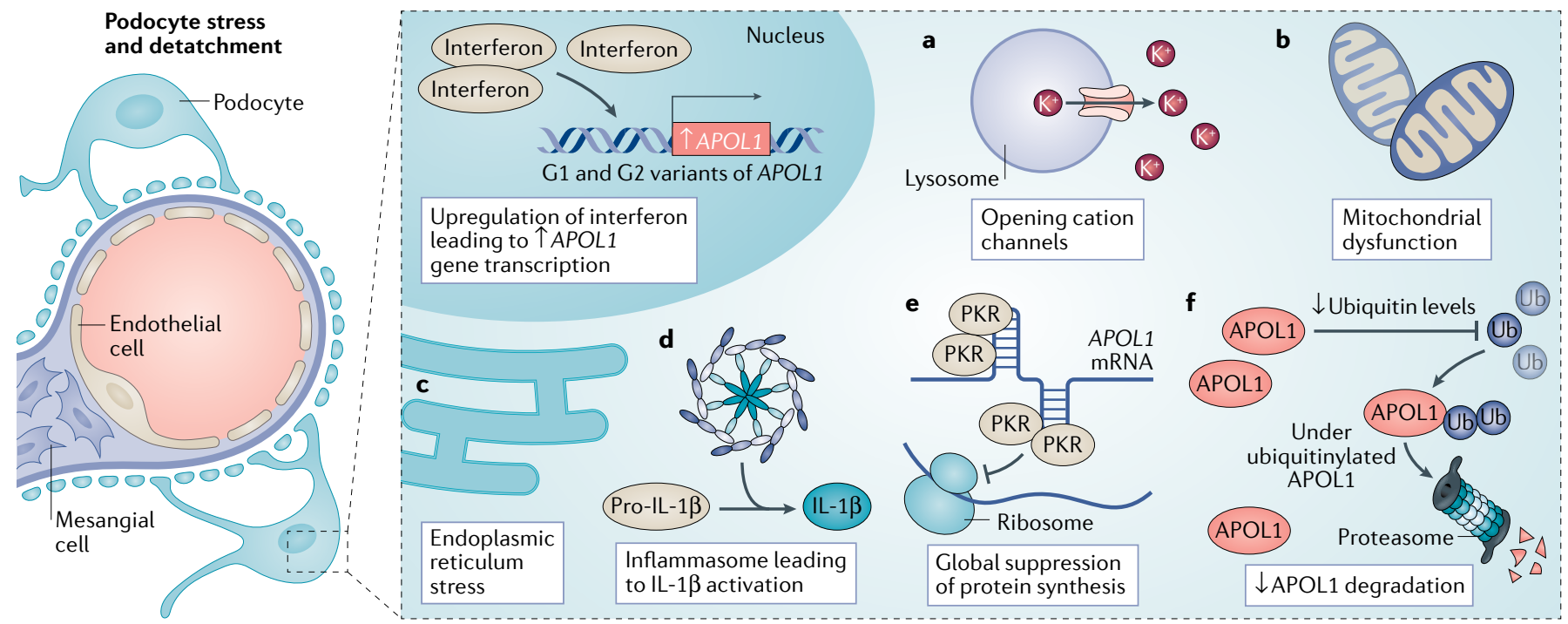

Fig. 3 | Potential mechanisms of APOL1-associated kidney disease. APOL1 is expressed in an interferon-dependent manner, and high-interferon states might result from the actions of a 'second hit', such as HIV infection. Several pathways have been implicated in the pathogenic response to APOL1 risk variants. For example, APOL1 variants may be associated with enhanced opening of cation channels, which compromises cell function (a), or may promote mitochondrial dysfunction through activation of the mitochondrial permeability transition pore (b). Alternatively, APOL1 variants may induce endoplasmic reticulum stress (c) and/or activate the NLRP3 inflammasome to generate active IL-1 $\beta$ (d). Other studies have demonstrated that APOL1 risk variants have stretches of double-stranded RNA, to which protein kinase R binds, inducing its autophosphorylation and suppressing of protein synthesis (e). Finally, APOL1 variants may reduce ubiquitin levels, prolonging the retention of intracellular proteins, including APOL1 itself (f). Although the relative importance of each of these injury pathways remains uncertain, the end result is damage to particular kidney cells, particularly the glomerular podocyte, resulting in dysfunction and in some cases, cell death and/or loss. IL-1 $\beta$, interleukin-1 $\beta$; PKR, protein kinase R; Ub, ubiquitin.
APOL1 and lysosomal dysfunction. In 2007, researchers showed that APOL1 kills trypanosomes by forming pores in the trypanosome lysosomal membrane, to which APOL1 traffics following uptake into the parasite ${ }^{103}$. Using endocytosis, the parasite takes up APOL1 in complex with HDL, from which the parasite obtains lipids and iron ${ }^{20}$. The structure of the $\mathrm{pH}$-sensitive membrane-addressing domain of APOL1 contains a hairpin region that connects two $a$-helices. This structural element undergoes a conformational change in the acidic environment of the lysosome, which allows insertion of APOL1 into the lysosome membrane and activation of the APOL1 pore-forming domain. APOL1-mediated pore formation disrupts the lysosomal membrane, triggering an uncontrolled influx of ions from the cytoplasm into the lysosome. This process leads to osmotic swelling of the lysosome and eventual trypanosomal death ${ }^{25,104,105}$.

In 2003, a carboxy-terminal $a$-helical domain was identified in APOL1, which interacts with the aminoterminal of SRA in T. $b$. rhodesiense (FIG. 1a). As described earlier, this SRA domain confers resistance to APOL1, eluding host defences and enabling the trypanosome to cause human disease ${ }^{39}$. A subsequent study showed that interaction with SRA altered the intracellular trafficking of APOL1, resulting in its localization to SRA-containing cytoplasmic vesicles instead of to the lysosomes, thereby inhibiting membrane toxicity in the parasite ${ }^{106}$. Additional studies of the C-terminal helix of APOL1 confirmed that interaction of the SRA domain with this region of APOL1 inhibits APOL1 toxicity by preventing pore-forming activity, thus protecting the trypanosome ${ }^{107}$.
Of note, APOL1 causes lysosomal dysfunction in cultured human kidney cells as well as in parasites. The APOL1 G1 and G2 variants decrease the number of lysosomes in podocytes, which results in leakage of lysosomal enzymes into the cytoplasm ${ }^{108}$. Over-expression of the APOL1 risk variants in cultured podocytes is associated with podocyte lysosomal swelling; this lysosomal dysfunction might occur through the functional downregulation of mechanistic target of rapamycin $(\mathrm{mTOR})^{109}$.

APOL1 as an ion channel. As described above, APOL1 acts as an ion channel in the lysosomal membranes of parasites and human cells, causing swelling and cell death. However, various studies have reported conflicting results with regard to the anionic versus cationic activity of these channels ${ }^{110}$. An initial report in 2005 showed that APOL1 targets the lysosomal lipid bilayer membrane, causing depolarization. This process was followed by an influx of chloride (with an observed chloride-to-potassium permeability ratio of 3.2 to 1 ), which was greater at $\mathrm{pH} 5$ than at neutral $\mathrm{pH}^{104}$, prompting these researchers to later propose that APOL1 acts as an anionic channel in the intracellular membranes of trypanosomes ${ }^{105}$.

In 2009, further support was provided for anion channel activity of APOL1 as a function of the trypanosome lytic factors ${ }^{104}$. Another study demonstrated the ability of a chloride-channel blocking agent to prevent podocyte swelling induced by APOL1 variants ${ }^{108}$. A subsequent study demonstrated that full-length APOL1 purified from human serum induces pores in phospholipid vesicles (liposomes), and that these pores allow 
Table 1 | Possible mechanisms of APOL1-associated glomerular injury

\begin{tabular}{|c|c|c|c|}
\hline Mechanism & $\begin{array}{l}\text { Evidence } \\
\text { in cell } \\
\text { culture }\end{array}$ & $\begin{array}{l}\text { Evidence } \\
\text { in mouse } \\
\text { models }\end{array}$ & $\begin{array}{l}\text { Evidence in } \\
\text { human tissues } \\
\text { or samples }\end{array}$ \\
\hline Increased APOL1 variant RNA expression & Yes & Yes & Yes \\
\hline Reduced APOL1 degradation & No & Yes & ND \\
\hline Inflammasome activation & No & Yes & ND \\
\hline Alterations in the actin cytoskeleton & Yes & Yes & Yes \\
\hline Increased membrane cation channel flux & Yes & ND & ND \\
\hline Mitochondrial dysfunction & Yes & ND & ND \\
\hline $\begin{array}{l}\text { Endoplasmic reticulum stress, blockade } \\
\text { of autophagic flux }\end{array}$ & Yes & Yes & ND \\
\hline $\begin{array}{l}\text { Protein kinase R activation; global } \\
\text { reduction in protein synthesis }\end{array}$ & Yes & Yes & ND \\
\hline Increased production of interleukin- $1 \beta$ & Yes & Yes & ND \\
\hline $\begin{array}{l}\text { Promotion of suPAR-dependent integrin } \\
\text { activation }\end{array}$ & Yes & Yes & Yes \\
\hline Intracellular cholesterol accumulation & Yes & Yes & ND \\
\hline Inhibition of APOL3 function & Yes & No & ND \\
\hline
\end{tabular}

$\mathrm{ND}$, not determined; suPAR, soluble urokinase plasminogen activator receptor.

passage of calcein, a 623 Da negatively charged molecule at neutral $\mathrm{pH}$. APOL1 channel activity was found to have three requirements that allow APOL1 to associate with pore-forming vesicles: low $\mathrm{pH}$, presence of negatively charged phospholipids in vesicle membranes and low ionic strength ${ }^{108}$.

In agreement with these findings, a 2005 study reported that APOL1 forms distinct anion-selective pores in unilamellar vesicular membranes, thereby promoting chloride influx. The researchers speculated that the passive entry of chloride through anion-selective channels is facilitated by the initial influx of extracellular sodium down its concentration gradient. The resulting osmotic imbalance leads to the passive entry of water to the cell, which promotes cell swelling and trypanosome lysis ${ }^{111}$.

However, a 2015 study reported quite different results, showing that APOL1-mediated trypanosome lysis requires an acidic $\mathrm{pH}$ for the first steps, in which APOL1 interacts with and inserts into vacuolar lipid bilayers. The researchers reported that APOL1 is subsequently trafficked to the plasma membrane, where it is exposed to a non-acidic $\mathrm{pH}$, allowing APOL1 to open $\mathrm{pH}$-sensitive non-selective cationic channels, depolarizing the trypanosome membrane and killing the trypanosome ${ }^{112}$. A subsequent publication supported the cation-selective nature of these ion channels by showing that mammalian cells expressing APOL1 risk variants have increased non-selective cation permeability and promote a net efflux of intracellular potassium through plasma membranes ${ }^{113}$. More recently, a population of plasma membrane cation channels for sodium and calcium were noted to be selectively expressed in HEK293 cells expressing APOL1 risk variants ${ }^{114}$.

These apparently contradictory findings may be explained by the suggestion that APOL1 ion-channel selectivity is $\mathrm{pH}$ switchable. At $\mathrm{pH} 5$, APOL1 may promote chloride permeability through anionic channels; at neutral $\mathrm{pH}$, it facilitates cationic (potassium) permeability, whereas it has almost no channel activity in a basic environment. Permeability to both ions requires negatively charged phospholipids for maximal activity. Moreover, potassium channels require calcium ions for proper function. This $\mathrm{pH}$-switchable ion-selective permeability may explain both the anionic and cationic channel activity previously reported for APOL1 in intracellular and plasma membrane environments, respectively, as the differences could be due to $\mathrm{pH}$ differences in the various intracellular compartments under particular experimental conditions ${ }^{115}$.

APOL1 and mitochondrial dysfunction. In addition to lysosomal toxicity, APOL1 is now understood to induce lysosome-mediated mitochondrial permeabilization through the actions of the $\mathrm{N}$-terminal pore-forming domain $^{116}$. The mechanisms by which APOL1 is transported to various organelles in the cell are unknown and deserve further investigation. However, acidic $\mathrm{pH}$, which is characteristic of the lysosomal-endocytic system, is required for the insertion of APOL1 into membranes, which in turn is crucial for transporting the protein to other organelles, including mitochondria. In the trypanosome, mitochondrial membrane permeabilization causes the release of mitochondrial, but not cytoplasmic, endonuclease G. This enzyme then traffics to the trypanosomal nucleus and causes nuclear changes, including condensation of chromatin into heterochromatin and DNA fragmentation. Mitochondrial permeabilization is induced by the depolarization that results from lysosomal membrane permeabilization and, therefore, the mitochondrial changes are not expected to occur in the absence of lysosomal toxicity. Moreover, knockdown of endonuclease $\mathrm{G}$ is associated with inhibition of trypanolysis, regardless of coexisting mitochondrial and lysosomal permeabilization ${ }^{116}$.

APOL1-induced mitochondrial dysfunction may contribute to APOL1-associated kidney disease. Studies in HEK293 cells stably transfected with doxycyclineinducible APOL1 G0, G1 and G2 alleles demonstrated localization of APOL1 to mitochondria. The induction of APOL1 G1 and G2 expression led to a marked reduction in maximum oxygen consumption rate and respiratory reserve capacity compared with that in APOL1 G0 variant-expressing cells. Impaired mitochondrial function occurred before reductions in intracellular potassium or cell viability, implying that mitochondrial dysfunction is mechanistically upstream of alterations in potassium flux ${ }^{117}$.

Permeabilization of mitochondria and the consequent depletion of energy compromises $\mathrm{Na}-\mathrm{K}$ ATPase function in the cell membrane, causing a subsequent loss of transmembrane electrical potential and inducing chloride and water influx, and resulting in cell swelling and death, as shown by Ma et al. ${ }^{117}$. HEK293 cells that express the APOL1 G1 and G2 variants show markedly lower cell viability than APOL1-G0-expressing cells. Cells that express the risk variants also show reduced synthesis of the metabolic cofactor nicotinamide adenine dinucleotide $\left(\mathrm{NAD}^{+}\right)$, due at least in part to downregulation of the gene encoding nicotinate phosphoribosyltransferase (NAPRT) a key enzyme in one of the pathways of $\mathrm{NAD}^{+}$synthesis. 
This downregulation of NAPRT and $\mathrm{NAD}^{+}$synthesis would be expected to compromise mitochondrial function $^{117}$.

Granado and colleagues evaluated the effect of APOL1 on mitochondrial dysfunction in podocyte cell lines, showing that localization of APOL1 to the endoplasmic reticulum and mitochondrial membrane was independent of the APOL1 N-terminal signal peptide. The authors also showed that the risk variants were associated with activation of stress-induced and AMP-activated protein kinases, reduced intracellular potassium levels and mitochondrial respiration rates, resulting in a subsequent decline in cell viability ${ }^{118}$.

Shah et al. showed that although both APOL1 wild type and risk variants are trafficked to the mitochondria via an as yet unidentified sequence in the $\mathrm{N}$-terminal protein domain, only APOL1 risk variants form higher-order oligomers inside the mitochondria and activate pore opening, leading to cell toxicity ${ }^{119}$. Effects on the tricarboxylic acid cycle, increased fatty acid oxidation and decreased redox homeostasis lead to impairment of mitochondrial membrane respiratory chain function, resulting in decreased mitochondrial membrane potential and cell toxicity ${ }^{120}$.

The presence of APOL1 in the cell cytosol has also been demonstrated by APOL1 antibody labelling. Clusters of APOL1 protein have been found in close proximity to mitochondria. Another member of the APOL family, APOL3, colocalizes with APOL1. Interaction of APOL1 with APOL3 decreases binding of APOL3 to neuronal calcium sensor 1, resulting in reduced PI4KB activity and dysregulation of actomyosin. These effects alter organellar trafficking, including mitochondrial fission, further linking APOL1 with mitochondrial dysfunction ${ }^{121}$.

Endoplasmic reticulum localization. Using cultured tubular epithelial HEK293 cells, Chun and colleagues demonstrated that APOL1 risk variants predominantly localize to the endoplasmic reticulum, whereas wild type APOL1 localizes to lipid droplets. Moreover, treatment of cells to promote the formation of lipid droplets shifted the localization of the G1 and G2 variants from the endoplasmic reticulum to lipid droplets. This shift was associated with a reduction in autophagic flux and cytotoxicity, suggesting that increasing lipid droplet localization of APOL1 risk variants might represent a means of decreasing cell cytotoxicity ${ }^{26}$.

More recently, use of a new, highly specific APOL1 antibody has demonstrated that apart from its expression in the plasma membrane, APOL1 in podocytes seems to be largely confined to the endoplasmic reticulum, and to a lesser degree the Golgi, and was absent from mitochondria. Moreover, most APOL1 was shown to localize to the inner luminal surface of the endoplasmic reticulum membrane, whereas APOL2 was found on the outer cytoplasmic surface and was thus absent from the cell surface ${ }^{122}$. Topology mapping of serum and podocytes showed that the $\mathrm{N}$-terminal pore-forming domain and C-terminal SRA protein-binding domain are exposed in membrane-bound, podocyte-expressed APOL1, whereas the membrane-addressing domain is exposed in serum APOL ${ }^{123}$. This finding implies that contextual differences in APOL1 topology (that is, in circulating versus membrane-bound APOL1) may mediate its selective toxicity.

APOL1 and inflammatory pathways. In 2016, an analysis of African-Americans with kidney disease enrolled in the Nephrotic Syndrome Study Network (NEPTUNE) study demonstrated an up-regulation of transcripts encoding the inflammatory cytokines CXCL9 and CXCL11 in kidney biopsy samples from individuals with APOL1 risk variants, compared with levels in other individuals with similar histological diagnoses ${ }^{124}$. These findings were reproduced in a study of transgenic mice, which showed that expression of the risk variants led to inflammation-mediated podocyte death and glomerular scarring ${ }^{72}$.

Ryu and colleagues reported that macrophages isolated from APOL1 G1 and G2 transgenic mice with hypertensive kidney injury demonstrated increased cholesterol accumulation compared with macrophages from APOL1 G0 transgenic mice, possibly through decreased expression of cholesterol efflux transporters. These findings suggest that macrophage foam cell formation might contribute to a pathway of injury and inflammation in carriers of the APOL1 risk alleles ${ }^{125}$.

Toll-like receptors (TLRs) are a component of the innate immune system. These receptors are expressed not only by macrophages and dendritic cells but also by podocytes, and function to recognize microbial macromolecules. The expression of APOL family members, particularly APOL1 and APOL3, is increased by TLR3 activation, and these APOL1 proteins mediate podocyte cell death resulting from poly (I:C)-induced TLR3 signalling. Thus, blocking TLR signalling has downstream implications on APOL1 expression levels and related toxicities. In addition, Uzureau and colleagues ${ }^{121}$ have shown that APOL1 risk variants demonstrate increased binding to APOL3, preventing APOL3-mediated activation of Golgi PI4KB - a kinase involved in podocyte actomyosin organization and ultimately resulting in podocyte impairment.

The NLRP3 inflammasome is a multiprotein complex that also forms part of the innate immune system and acts to activate various inflammatory proteins. Using cultured podocytes, Jha and colleagues demonstrated that the presence of two APOL1 risk alleles led to enhanced entry of $\mathrm{K}+$, activation of the NLRP3 inflammasome and increased production of IL- $1 \beta$ and IL-18 (REF. ${ }^{126}$ ). These emerging findings highlight important roles for APOL1 risk variants in diverse inflammation pathways; further studies are necessary to parse the specific pathways in relevant cell types and tissues.

APOL1 and autophagic cell death. APOL1 is a member of the Bcl-2 homology domain 3 (BH3) family, which is involved in programmed, autophagic cell death in a spectrum of cell types and a broad spectrum of disease states. The demonstration that deletion of the BCL2- homology 3 (BH3) domain within the pore-forming domain of APOL1 leads to loss of APOL1 cell toxicity ${ }^{127}$ led researchers to speculate that the $\mathrm{BH} 3$ domain is the critical 
effector in APOL1-mediated cell death. However, despite supporting findings from studies of Xenopus oocytes ${ }^{128}$, mutation analyses demonstrated that the $\mathrm{BH} 3$ region is dispensable for the cell toxicity and trypanolytic activity of APOL1. Moreover, BH3 domain-mediated toxicity of APOL1 can be rescued by the co-expression of particular anti-apoptotic proteins from the BCL2 family ${ }^{128}$.

Activation of protein kinase R. Protein kinase R (PKR) is activated by double-stranded RNA, which is present in cells during some viral infections. Activation of PKR leads to suppression of protein synthesis, which serves to limit viral replication at the cost of cell stress or cell death. APOL1 risk variant RNAs have sufficient stretches of double-stranded RNA to activate PKR in contrast to G0, which does not activate PKR. Activation of PKR inhibits protein synthesis and induces glomerular disease in transgenic mice carrying APOL1 risk variants, but no PKR inhibitors are currently available for clinical investigation $^{129}$.

UBD expression and APOL1 stability. A 2018 study demonstrated a potential link between alterations in the ubiquitin-proteasome system and APOL1 nephropathy. Findings from admixture mapping and in vitro studies support a model in which ubiquitin D (UBD) a ubiquitin-like modifier protein that targets proteins to the proteosome for degradation - mitigates APOL1-mediated toxicity by targeting it for destruction. Zhang and colleagues showed that Individuals who had lower expression of UBD and who carried two APOL1 risk alleles were more prone to developing APOL1 nephropathy than individuals with normal UBD levels ${ }^{130}$.

\section{Therapeutic approaches}

Current therapies employed for APOL1 nephropathies are non-specific and often fail to prevent progressive loss of kidney function. As noted above, the pathophysiology of APOL1 variant-induced cell dysfunction is complex. (FIG. 3). Further studies are needed to confirm that in patients with APOL1 nephropathies, these cellular pathways are dysregulated and essential to kidney injury. If this is confirmed, a number of therapeutic approaches may be possible, as discussed below.

APOL1 expression. The observations that the chimpanzee, our nearest extant animal kin, lacks the APOL1 gene $^{131}$ and that one individual was found, after becoming infected with the unusual pathogen Trypanosoma evansi, to lack the APOL1 gene but was otherwise healthy, suggest that APOL1 is not essential for normal growth, development and function ${ }^{132}$. Thus, reducing APOL1 expression could be a safe approach (outside of regions with endemic African trypanosomiasis) and might be an effective approach to treating APOL1 nephropathies. As discussed above, evidence overall suggests that plasma APOL1 does not affect the kidney and, thus, therapeutics that suppress the hepatic production of APOL1 will likely not be successful. Instead, APOL1-targeted therapeutics will need to target the kidney.

Two clinical studies have been launched recently to test novel compounds against APOL1 levels and activity.
One study, by Ionis Pharmaceuticals (Carlsbad, CA, USA), used antisense oligonucleotide analogues to reduce $A P O L 1$ RNA expression in APOL1 transgenic mice ${ }^{133}$, reducing APOL1 expression in kidneys and ameliorating proteinuria ${ }^{133}$. The agent has been licensed to AstraZeneca (Cambridge, UK), which conducted a first-in-human, single ascending dose, phase I study ${ }^{134}$ ) to evaluate the safety and assess the pharmacokinetics of escalating single doses of a subcutaneously administered antisense oligonucleotide (AZD2373), directed against APOL1, in 48 healthy men of African descent. This study has been terminated, seemingly as the sponsor deemed that sufficient data were available to proceed with further studies and higher doses were associated with injection site reactions.

A phase II study sponsored by Vertex Pharmaceuticals (Boston, MA, USA) is testing an oral small molecule inhibitor of APOL1 activity, VX-147, in patients with APOL1-associated FSGS ${ }^{135}$. News of these trials of novel agents, admittedly of unknown efficacy, brings hope to patients with diseases that too often progress to kidney failure.

Inflammatory pathways. The overexpression of APOL1 risk variants causes kidney injury in various experimental models. As described earlier, APOL1 nephropathies in humans generally require a second factor. Specifically, high-interferon states, such as those observed in viral infections and systemic lupus erythematosus, have been associated with increased expression of APOL1 $\left(\mathrm{REFS}^{136,137}\right)$ and APOL1 nephropathies. Kidney disease has also been reported in individuals with APOL1 risk variants after receiving therapeutic interferon ${ }^{138}$. Thus, glomerular and microvascular injury associated with APOL1 variants may be diminished using therapeutic approaches affecting interferon-related pathways. A 2019 study showed that nucleosomal double-stranded DNA induces APOL1 expression through both interferonindependent and -dependent signalling pathways. Hence, it is plausible that interferon antagonism can be used to reduce APOL1 expression in podocytes in response to nucleosomal double-stranded DNA ${ }^{139}$.

Rontazilumab, sifalimumab and anifrolumab are monoclonal anti-interferon antibodies that have been tested in clinical trials of systemic lupus erythematosus and Crohn disease, although none has been approved by the FDA. Their ability to neutralize interferon makes them suitable candidates for APOL1-related disorders $^{140-142}$.

Interferon is produced in response to TLR activation and as mentioned earlier, members of the APOL family can regulate cell death triggered by TLR3 signalling downstream of poly (I:C) ${ }^{121}$. Thus, another approach to APOL1 nephropathy is to target the TLR pathway ${ }^{138}$. Eritoran, a lipid A derivative, and the small molecule inhibitor resatorvid, antagonize TLR4 signalling, and have proposed therapeutic roles as anti-inflammatory agents, in severe sepsis, and as adjuvants for chemotherapy and neuroprotection, although they are not yet approved by the $\mathrm{FDA}^{143}$. Pathways downstream of TLR3 have also been targeted in the context of rheumatoid $\operatorname{arthritis}^{144}$. 
IL-1 is produced by podocytes and contributes to proinflammatory pathways in APOL1 nephropathies, likely as a consequence of NLRP3 inflammasome activation $^{126}$. Three IL-1 antagonists are licensed by the FDA for the treatment of autoinflammatory disorders: anakinra, canakinumab and rilonacept ${ }^{145}$. Such agents may demonstrate therapeutic potential in the context of APOL1 nephropathy.

Membrane channels. As described earlier, uncertainty exists as to whether APOL1 affects the function of cationic or anionic channels, or perhaps both ${ }^{112,146}$. Several therapeutics are available that affect either type of channel, including agents that interact directly with the channels, such as direct potassium channel inhibitors, and those that modulate upstream or downstream components, such as mitogen-activated protein (MAP) kinase inhibitors ${ }^{147,148}$. Further studies are required to develop therapies that directly inhibit pore assembly or ion flux induced by APOL1 variants.

Mitochondrial dysfunction. Various therapies for mitochondrial dysfunction are available. The least toxic is probably co-enzyme 10, which facilitates electron flow in the mitochondrial electron chain. Whether such agents will demonstrate therapeutic benefit in APOL1 nephropathies remains to be determined.

Endoplasmic reticulum stress. As described above, APOL1 risk variants are associated with the endoplasmic reticulum. Use of a podocyte culture model demonstrated that APOL1 risk variants induce the expression of $78-\mathrm{kDa}$ glucose-regulated protein (GRP78) and induce phosphorylation of eukaryotic translation initiation factor 1 (eIF1) - markers of endoplasmic reticulum stress, with recovery of podocyte protein expression upon treatment with inhibitors of endoplasmic reticulum stress ${ }^{149}$. Podocyte endoplasmic reticulum stress is also associated with other glomerular diseases, including diabetic nephropathy ${ }^{150}$. Factors that promote endoplasmic reticulum stress and have the potential to exacerbate CKD include tissue hypoxia, oxidative stress, and chronic inflammation. Numerous agents can attenuate endoplasmic reticulum stress, including glucocorticoids, endoplasmic reticulum chaperones such as tauroursodeoxycholic acid and trehalose, and molecular chaperone modulators, which promote proper protein folding. These agents have been studied in other conditions, such as cholestatic liver disease (for which tauroursodeoxycholic acid is FDA-approved), cystic fibrosis and Gaucher disease, and as a food additive (trehalose). However, further studies are needed to confirm whether endoplasmic reticulum stress is induced by the APOL1 risk variants in relevant model systems and particularly in human patients.

\section{Psychosocial issues}

African Americans have a long history of social and economic discrimination, extending to inequity in medical care access and delivery and vulnerability in medical research. Notable and disturbing examples include the notorious Tuskegee experiments, in which African Americans were denied treatment for syphilis, and have contributed to a distrust of medical care and medical research in particular ${ }^{151}$. In 2020, the "Black Lives Matter" movement focused the world's attention on these long-festering social problems in the United States and elsewhere. Against this background, groups of researchers have undertaken interviews with African Americans to obtain their insights into the best approaches to informing this population of the risks of APOL1 variants and to identify optimal approaches to involving African Americans in research, trial design, in clinical trials, the dissemination of clinical trial results and in the development of screening and treatment recommendations, as these aspects evolve ${ }^{152,153}$.

\section{Conclusions and future perspectives}

That APOL1 renal risk variants strongly associate with kidney disease is now clear. However, important knowledge gaps remain in our understanding of APOL1-mediated kidney pathogenesis, the absolute risk attributable to APOL1 variants and the course of APOL1 nephropathies.

A growing body of research has provided insights into various cellular pathways that are stimulated by the APOL1 risk variants in cell and animal studies; however, the exact pathways that are relevant in specific patients or at specific disease stages is unclear. In line with this knowledge gap, we currently do not know what proportion of APOL1 risk variant carriers will develop kidney disease. We do not know why only some individuals with APOL1 risk variants develop kidney disease; the contributing factors, beyond interferons; and whether any protective factors - either genetic or environmental - exist.

In terms of the clinical presentation and course of APOL1 nephropathies, we do not know the extent to which the annual risk of incident kidney disease differs over the life course of these individuals; the extent to which APOL1 kidney disease first manifests as microalbuminuria, macroalbuminuria or reduced eGFR; and whether early detection by proteinuria screening, followed by standard therapies (such as anti-hypertensives, salt restriction, renin-angiotensin-aldosterone system antagonists and/or low-dose thiazide diuretics) or experimental therapies (such as those that target APOL1 variants or the affected pathways) might improve kidney outcomes. Other areas that require investigation are the degree of CKD risk for potential kidney donors with APOL1 high-risk genotypes and the psychological effects of providing information about APOL1 risk status to healthy individuals with $A P O L 1$ risk variants.

The answers to these outstanding questions will likely be forthcoming in the next few years. Prospective studies of individuals with APOL1 risk variants and normal kidney function are underway, as are studies aimed at addressing the impact of receiving APOL1 genetic test results. Hopes are also high for new therapeutic options for patients with APOL1 nephropathies, particularly for a novel agent currently undergoing a clinical trial. Moreover, improved understanding of the molecular and cellular mechanisms of APOL1-associated kidney disease will likely identify novel therapeutic avenues for investigation.

Published online 25 February 2022 
1. Genovese, G. et al. Association of trypanolytic ApoL1 variants with kidney disease in African Americans. Science 329, 841-845 (2010).

2. Tzur, S. et al. Missense mutations in the APOL1 gene are highly associated with end stage kidney disease risk previously attributed to the MYH9 gene. Hum. Genet. 128, 345-350 (2010).

3. The Churchill Society London. The Churchill Society http://www.churchill-society-london.org.uk/EndoBegn. html (1942).

4. Nadkarni, G. N. et al. Worldwide frequencies of APOL1 renal risk variants. N. Engl. J. Med 379 , 2571-2572 (2018)

5. Micheletti, S. J. et al. Genetic consequences of the transatlantic slave trade in the Americas. Am. J. Hum. Genet. 107, 265-277 (2020).

6. Bruggeman L. A., O'Toole, J. F \& Sedor, J. R. Identifying the intracellular function of APOL1. J. Am. Soc. Nephrol. 28, 1008-1011 (2017).

7. Limou, S., Nelson, G. W., Kopp, J. B. \& Winkler, C. A APOL1 kidney risk alleles: population genetics and disease associations. Adv. Chronic Kidney Dis. 21 426-433 (2014)

8. Johnstone, D. B. et al. APOL1 null alleles from a rural village in India do not correlate with glomerulosclerosis PLoS One 7, e51546 (2012).

9. Dummer, P. D. et al. APOL1 kidney disease risk variants: an evolving landscape. Semin. Nephrol. 35, 222-236 (2015)

10. Bruggeman, L. A., O’Toole, J. F. \& Sedor, J. R. APOL1 polymorphisms and kidney disease: loss-of-function or gain-of-function? Am. J. Physiol. Renal Physiol. 316 F1-F8 (2019)

11. Friedman, D. J. A brief history of APOL1: a gene evolving. Semin. Nephrol. 37, 508-513 (2017).

12. Hughson, M. D. et al. Risk variants independently associated with early cardiovascular disease death. Kidney Int. Rep. 3, 89-98 (2018)

13. Kopp, J. B. et al. APOL1 genetic variants in focal segmental glomerulosclerosis and HIV-associated nephropathy. J. Am. Soc. Nephrol. 22, 21 29-2137 (2011).

14. Reidy, K. J. et al. Fetal-not maternal-APOL1 genotype associated with risk for preeclampsia in those with African ancestry. Am. J. Hum. Genet. 103, 367-376 (2018)

15. Reeves-Daniel, A. M. et al. The APOL1 gene and allograft survival after kidney transplantation. Am. J. Transpl. 11, 1025-1030 (2011).

16. Duchateau, P. N. et al. Apolipoprotein L, a new human high density lipoprotein apolipoprotein expressed by the pancreas. Identification, cloning, characterization, and plasma distribution of apolipoprotein L. J. Biol. Chem. 272, 25576-25582 (1997).

17. Duchateau, P. N., Pullinger, C. R., Cho, M. H., Eng, C. \& Kane, J. P. Apolipoprotein L gene family: tissue-specific expression, splicing, promoter regions; discovery of a new gene. J. Lipid Res. 42, 620-630 (2001).

18. Page, N. M., Butlin, D. J., Lomthaisong, K. \& Lowry, P. J. The human apolipoprotein L gene cluster: identification, classification, and sites of distribution. Genomics 74, 71-78 (2001).

19. Glazko, G. V. \& Nei, M. Estimation of divergence times for major lineages of primate species. Mol. Biol. Evol. 20, 424-434 (2003)

20. Smith, E. E. \& Malik, H. S. The apolipoprotein L family of programmed cell death and immunity genes rapidly evolved in primates at discrete sites of host-pathogen interactions. Genome Res. 19, 850-858 (2009).

21. Kopp, J. B. et al. MYH9 is a major-effect risk gene for focal segmental glomerulosclerosis. Nat. Genet. $\mathbf{4 0}$ 1175-1184 (2008)

22. Kao, W. H. et al. MYH9 is associated with nondiabetic end-stage renal disease in African Americans. Nat. Genet. 40, 1185-1192 (2008).

23. Kasembeli, A. N. et al. APOL1 risk variants are strongly associated with HIV-associated nephropathy in Black South Africans. J. Am. Soc. Nephrol. 26, 2882-2890 (2015)

24. O'Toole, J. F. et al. ApoL1 overexpression drives variant-independent cytotoxicity. J. Am. Soc. Nephrol. 29, 869-879 (2018)

25. Limou, S., Dummer, P. D., Nelson, G. W. Kopp, J. B. \& Winkler, C. A. APOL1 toxin, innate immunity, and kidney injury. Kidney Int. 88, 28-34 (2015)

26. Chun, J et al. Recruitment of APOL1 kidney disease risk variants to lipid droplets attenuates cell toxicity. Proc. Natl Acad. Sci. USA 116, 3712-3721 (2019).

27. Shah, S. S. et al. APOL1 kidney risk variants induce cell death via mitochondrial translocation and opening of the mitochondrial permeability transition pore. J. Am. Soc. Nephrol. 30, 2355-2368 (2019).

28. Bruno, J. \& Edwards, J. C. Kidney disease-associated variants of Apolipoprotein L1 show gain-of-function in cation channel activity. J. Biol. Chem. 296, 100238 (2021).

29. Lannon, H. et al. Apolipoprotein L1 (APOL1) risk variant toxicity depends on the haplotype background. Kidney Int. 96, 1303-1307 (2019).

30. Datta, S. et al. Kidney disease-associated APOL variants have dose-dependent, dominant toxic gain-of-function. J. Am. Soc. Nephrol. 31, 2083-2096 (2020).

31. Thomson, R. et al. Evolution of the primate trypanolytic factor APOL1. Proc. Natl Acad. Sci. USA 111, E2130-E2139 (2014).

32. Limou, S. et al. Sequencing rare and common APOL1 coding variants to determine kidney disease risk. Kidney Int. 88, 754-763 (2015)

33. Wasser, W. G. et al. Population genetics of chronic kidney disease: the evolving story of APOL1. J. Nephrol. 25, 603-618 (2012).

34. Ko, W. Y. et al. Identifying Darwinian selection acting on different human APOL1 variants among diverse African populations. Am. J. Hum. Genet. 93, 54-66 (2013).

35. Freedman, B. I., Limou, S., Ma, L. \& Kopp, J. B. APOL1-associated nephropathy: a key contributor to racial disparities in CKD. Am. J. Kidney Dis. 72, S8-S16 (2018).

36. Purswani, M. U. et al. Brief report: APOL1 renal risk variants are associated with chronic kidney disease in children and youth with perinatal HIV infection. J. Acquir. Immune Defic. Syndr. 73, 63-68 (2016).

37. Giordani, F., Morrison, L. J., Rowan, T. G., De Koning, H. P. \& Barrett, M. P. The animal trypanosomiases and their chemotherapy: a review. Parasitology 143, 1862-1889 (2016)

38. Bishop, J. R., Shimamura, M. \& Hajduk, S. L. Insigh into the mechanism of trypanosome lytic factor-1 killing of Trypanosoma brucei brucei. Mol. Biochem. Parasitol. 118, 33-40 (2001).

39. Vanhamme, L. et al. Apolipoprotein L-I is the trypanosome lytic factor of human serum. Nature 422, 83-87 (2003).

40. Shiflett, A. M., Bishop, J. R., Pahwa, A. \& Hajduk, S. L. Human high density lipoproteins are platforms for the assembly of multi-component innate immune complexes. J. Biol. Chem. 280, 32578-32585 (2005).

41. Molina-Portela, M. P Samanovic, M \& Raper J. Distinct roles of apolipoprotein components within the trypanosome lytic factor complex revealed in a novel transgenic mouse model. J. Exp. Med. 205 1721-1728 (2008).

42. Cooper, A. et al. APOL1 renal risk variants have contrasting resistance and susceptibility associations with African trypanosomiasis. eLife 6, e25461 (2017).

43. Kaboré, J. W. et al. Candidate gene polymorphisms study between human African trypanosomiasis clinical phenotypes in Guinea. PLoS Negl. Trop. Dis. 11, e0005833 (2017).

44. Kimuda, M. P. et al. No evidence for association between APOL1 kidney disease risk alleles and human African trypanosomiasis in two Ugandan populations. PLoS Negl. Trop. Dis. 12, e0006300 (2018).

45. Chang, J. H. et al. Donor's APOL1 risk genotype and "second hits" associated with de novo collapsing glomerulopathy in deceased donor kidney transplant recipients: a report of 5 Cases. Am. J. Kidney Dis. 73 134-139 (2019)

46. Kopp, J. B., Heymann, J. \& Winkler, C. A. APOL1 renal risk variants: fertile soil for HIV-associated nephropathy. Semin. Nephrol. 37, 514-519 (2017).

47. An, P. et al. Impact of APOL1 genetic variants on HIV-1 infection and disease progression. Front. Immunol. 10, 53 (2019).

48. Blazer, A. D. \& Clancy, R. M. ApoL1 and the immune response of patients with systemic lupus erythematosus. Curr. Rheumatol. Rep. 19, 13 (2017).

49. Riella, C. et al. APOL1-associated kidney disease in Brazil. Kidney Int. Rep. 4, 923-929 (2019).

50. Peralta, C. A. et al. APOL1 genotype and race differences in incident albuminuria and renal function decline. J. Am. Soc. Nephrol. 27, 887-893 (2016).

51. Chen, T. K. et al. Risk variants and cardiovascular disease: results from the AASK (African American Study of Kidney Disease and Hypertension).
Arterioscler. Thromb. Vasc. Biol. 37, 1765-1769 (2017).

52. Freedman, B. I. \& Skorecki, K. Gene-gene and gene-environment interactions in apolipoprotein L1 gene-associated nephropathy. Clin. J. Am. Soc Nephrol. 9, 2006-2013 (2014).

53. Langefeld, C. D. et al. Genome-wide association studies suggest that APOL1-environment interactions more likely trigger kidney disease in African Americans with nondiabetic nephropathy than strong APOL1-second gene interactions. Kidney Int. 94 599-607 (2018)

54. Husain, S. A. $\&$ Chang, J. H. Searching for second hits for the development of APOL1-associated kidney disease. Kidney Int. Rep. 4, 911-913 (2019).

55. Ma, L., Divers, J. \& Freedman, B. I. Mechanisms of Injury in APOL1-associated kidney disease. Transplantation 103, 487-492 (2019).

56. Kudose, S. et al. Kidney biopsy findings in patients with COVID-19. J. Am. Soc. Nephrol. 31, 1959-1968 (2020)

57. Wu, H. et al. AKI and collapsing glomerulopathy associated with COVID-19 and APOL 1 high-risk genotype. J. Am. Soc. Nephrol. 31, 1688-1695 (2020).

58. Lazareth, H. et al. COVID-19-related collapsing glomerulopathy in a kidney transplant recipient Am. J. Kidney Dis. 76, 590-594 (2020).

59. Tedla, F. M. \& Yap, E. Apolipoprotein L1 and kidney transplantation. Curr. Opin. Organ. Transpl. 24, 97-102 (2019)

60. Mena-Cutierrez A. M. Reeves-Daniel, A. M Jay, C. L. \& Freedman, B. I. Practical considerations for APOL1 genotyping in the living kidney donor evaluation. Transplantation 104, 27-32 (2019)

61. Doshi, M. D. et al. Genotype and renal function of black living donors. J. Am. Soc. Nephrol. 29 , 1309-1316 (2018).

62. Lee, B. T. et al. The APOL1 genotype of African American kidney transplant recipients does not impact 5-year allograft survival. Am. J. Transpl. 12 1924-1928 (2012).

63. Zhang, Z. et al. Recipient APOL1 risk alleles associate with death-censored renal allograft survival and rejection episodes. J. Clin. Invest. 131, e 146643 (2021).

64. Kopp, J. B. \& Winkler, C. A. Genetic Testing for APOL1 genetic variants in clinical practice: finally starting to arrive. Clin. J. Am. Soc. Nephrol. 15, 126-128 (2019).

65. Locke, J. E. et al. Apolipoprotein L1 and chronic kidney disease risk in young potential living kidney donors. Ann. Surg. 267, 1161-1168 (2018)

66. Shah, S., Shapiro, R., Murphy, B. \& Menon, M. C. APOL1 high-risk genotypes and renal transplantation. Clin. Transpl. 33, e13582 (2019).

67. US National Library of Medicine. ClinicalTrials.gov https://clinicaltrials.gov/ct2/show/NCT03615235 (2021).

68. Ekulu, P. M. et al. Risk genotypes are associated with early kidney damage in children in Sub-Saharan Africa. Kidney Int. Rep. 4, 930-938 (2019).

69. Ross, M. J. New insights into APOL1 and kidney disease in African children and Brazilians living with end-stage kidney disease. Kidney Int. Rep. 4 908-910 (2019).

70. $\mathrm{Ng}, \mathrm{D}$. K. et al. APOL1-associated glomerular disease among African-American children: a collaboration of the Chronic Kidney Disease in Children (CKiD) and Nephrotic Syndrome Study Network (NEPTUNE) cohorts. Nephrol. Dial. Transpl. 32, 983-990 (2017).

71. Kozlitina, J. et al. Plasma levels of risk-variant APOL do not associate with renal disease in a populationbased cohort. J. Am. Soc. Nephrol. 27, 3204-3219 (2016).

72. Beckerman, P. et al. Transgenic expression of human APOL1 risk variants in podocytes induces kidney disease in mice. Nat. Med. 23, 429-438 (2017).

73. Madhavan, S. M. et al. APOL1 localization in normal kidney and nondiabetic kidney disease. J. Am. Soc. Nephrol. 22, 2119-2128 (2011)

74. Ma, L. et al. Localization of APOL1 protein and mRNA in the human kidney: nondiseased tissue, primary cells, and immortalized cell lines. J. Am. Soc. Nephrol. 26, 339-348 (2015)

75. Kotb, A. M. et al. Knockdown of ApoL1 in Zebrafish larvae affects the glomerular filtration barrier and the expression of nephrin. PLoS One 11, e0153768 (2016).

76. Weckerle, A. et al. Characterization of circulating APOL1 protein complexes in African Americans. J. Lipid Res. 57, 120-130 (2016). 
77. Shukha, K. et al. Most ApoL1 is secreted by the liver. J. Am. Soc. Nephrol. 28, 1079-1083 (2017).

78. Bruggeman, L. A. et al. APOL1-G0 or APOL1-G2 transgenic models develop preeclampsia but not kidney disease. J. Am. Soc. Nephrol. 27, 3600-3610 (2016).

79. Kumar, V. et al. Disruption of APOL1-miR193a axis induces disorganization of podocyte actin cytoskeleton. Sci. Rep. 9, 3582 (2019)

80. Hayek, S. S. et al. A tripartite complex of suPAR, APOL1 risk variants and $\alpha$. Nat. Med. 23, 945-953 (2017)

81. Anderson, B. R. et al. Correction: in vivo modeling implicates APOL1 in nephropathy: evidence for dominant negative effects and epistasis under anemic stress. PLoS Genet. 11, e1005459 (2015).

82. Anderson, B. R. et al. In vivo modeling implicates APOL1 in nephropathy: evidence for dominant negative effects and epistasis under anemic stress. PLoS Genet. 11, e 1005349 (2015).

83. Olabisi, O. et al. From man to fish: What can Zebrafish tell us about ApoL1 nephropathy? Clin. Nephrol. 86 114-118 (2016)

84. Kruzel-Davila, E. et al. APOL1-mediated cell injury involves disruption of conserved trafficking processes. J. Am. Soc. Nephrol. 28, 1117-1130 (2017).

85. Fu, Y. et al. APOL1-G1 in nephrocytes induces hypertrophy and accelerates cell death. J. Am. Soc. Nephrol. 28, 1106-1116 (2017)

86. Waitzman, J. S. \& Lin, J. Ten years in: APOL1 reaches beyond the kidney. Curr. Opin. Nephrol. Hypertens. 28, 375-382 (2019).

87. Bick, A. G. et al. Association of APOL1 risk alleles with cardiovascular disease in blacks in the million veteran program. Circulation 140, 1031-1040 (2019).

88. Mukamal, K. J. et al. APOL1 genotype, kidney and cardiovascular disease, and death in older adults. Arterioscler. Thromb. Vasc. Biol. 36, 398-403 (2016)

89. Hughson, M. D. et al. Risk alleles are associated with more severe arteriosclerosis in renal resistance vessels with aging and hypertension. Kidney Int. Rep. 1, 10-23 (2016).

90. Sumaili, E. K. et al. G1 is the major. Clin. Kidney J. 12 188-195 (2019)

91. Nadkarni, G. N. \& Coca, S. G. APOL1 and blood pressure changes in young adults. Kidney Int. $\mathbf{9 2}$ 793-795 (2017).

92. Akinyemi, R. et al. APOL1, CDKN2A/CDKN2B, and HDAC9 polymorphisms and small vessel ischemic stroke. Acta Neurol. Scand. 137, 133-141 (2018).

93. Franceschini, N. et al. Association of APOL1 with heart failure with preserved ejection fraction in postmenopausal African American women. JAMA Cardiol. 3, 71 2-720 (2018).

94. Chen, T. K. et al. Association between APOL1 genotypes and risk of cardiovascular disease in MESA (multi-ethnic study of atherosclerosis). J. Am. Heart Assoc. 6, e007199 (2017).

95. McLean, N. O., Robinson, T. W. \& Freedman, B. I. APOL1 gene kidney risk variants and cardiovascular disease: getting to the heart of the matter. $\mathrm{Am}$. J. Kidney Dis. 70, 281-289 (2017).

96. Freedman, B. I. et al. renal-risk variants do not associate with incident cardiovascular disease or mortality in the Systolic Blood Pressure Intervention Trial. Kidney Int. Rep. 2, 713-720 (2017).

97. Grams, M. E. et al. Kidney risk variants and cardiovascular disease: an individual participant data meta-analysis. J. Am. Soc. Nephrol. 30, 2027-2036 (2019).

98. Gutiérrez, O. M. et al. APOL1 nephropathy risk variants do not associate with subclinical atherosclerosis or left ventricular mass in middle-aged black adults. Kidney Int. 93, 727-732 (2018).

99. Parê, E. et al. Clinical risk factors for preeclampsia in the 21st century. Obstet. Gynecol. 124, 763-770 (2014).

100. Hong, X. et al. Joint associations of maternal-fetal APOL1 genotypes and maternal country of origin with preeclampsia risk. Am. J. Kidney Dis. 77, 879-88.e1 (2021).

101. Chaudhary, N. S. et al. Nephropathy risk alleles and risk of sepsis in blacks. Clin. J. Am. Soc. Nephrol. 14 1733-1740 (2019)

102. Ma, L. \& Freedman, B. I. Nephropathy risk variant associations with diseases beyond the kidney: APOL1 and sepsis. Clin. J. Am. Soc. Nephrol. 14, 1684-1686 (2019).

103. Vanhollebeke, B., Lecordier, L., Perez-Morga, D., Amiguet-Vercher, A. \& Pays, E. Human serum lyses Trypanosoma brucei by triggering uncontrolled swelling of the parasite lysosome. J. Eukaryot. Microbiol. 54, 448-451 (2007).

104. Perez-Morga, D. et al. Apolipoprotein L-I promotes trypanosome lysis by forming pores in lysosomal membranes. Science 309, 469-472 (2005).

105. Vanhollebeke, B. \& Pays, E. The function of apolipoproteins L. Cell Mol. Life Sci. 63, 1937-1944 (2006).

106. Shiflett, A. M. et al. African trypanosomes intracellular trafficking of host defense molecules. J. Eukaryot. Microbiol. 54, 18-21 (2007).

107. Lecordier L et al. C-terminal mutants of apolipoprotein L-I efficiently kill both Trypanosoma brucei brucei and Trypanosoma brucei rhodesiense. PLoS Pathog. 5, e1000685 (2009).

108. Lan, X. et al. APOL1 risk variants enhance podocyte necrosis through compromising lysosomal membrane permeability. Am. J. Physiol. Ren. Physiol. 307, F326-F336 (2014)

109. Kumar, V. \& Singhal, P. C. APOL1 and kidney cell function. Am. J. Physiol. Renal Physiol. 307, F463-F477 (2019)

110. Kruzel-Davila, E. \& Skorecki, K. Dilemmas and challenges in apolipoprotein L1 nephropathy research. Curr. Opin. Nephrol. Hypertens. 28, 77-86 (2019)

111. Molina-Portela, M. P., Lugli, E. B., Recio-Pinto, E. \& Raper, J. Trypanosome lytic factor, a subclass of high-density lipoprotein, forms cation-selective pores in membranes. Mol. Biochem. Parasitol. 144 218-226 (2005)

112. Thomson, R. \& Finkelstein, A. Human trypanolytic factor APOL1 forms $\mathrm{pH}$-gated cation-selective channels in planar lipid bilayers: relevance to trypanosome lysis. Proc. Natl Acad. Sci. USA 112 , 2894-2899 (2015).

113. Olabisi, O. A. et al. APOL1 kidney disease risk variants cause cytotoxicity by depleting cellular potassium and inducing stress-activated protein kinases. Proc. Natl Acad. Sci. USA 113, 830-837 (2016).

114. Giovinazzo, J. A. et al. Apolipoprotein L-1 renal risk variants form active channels at the plasma membrane driving cytotoxicity. eLife 9, e51185 (2020).

115. Bruno, J., Pozzi, N., Oliva, J. \& Edwards, J. C. Apolipoprotein $\mathrm{L} 1$ confers $\mathrm{pH}$-switchable ion permeability to phospholipid vesicles. J. Biol. Chem. 292, 18344-18353 (2017).

116. Vanwalleghem, G. et al. Coupling of lysosomal and mitochondrial membrane permeabilization in trypanolysis by APOL1. Nat. Commun. 6, 8078 (2015).

117. Ma, L. et al. Renal-risk variants induce mitochondrial dysfunction. J. Am. Soc. Nephrol. 28, 1093-1105 (2017).

118. Granado, D. et al. Intracellular APOL1 risk variants cause cytotoxicity accompanied by energy depletion. J. Am. Soc. Nephrol. 28, 3227-3238 (2017).

119. Shah, S. S. et al. APOL1 kidney risk variants induce cell death. J. Am. Soc. Nephrol. 30, 2355-2368 (2019).

120. Ma, L. et al. APOL1 risk variants impair multiple mitochondrial pathways in a metabolomics analysis. Kidney360 1, 1353-1362 (2020).

121. Uzureau, S. et al. APOL1 C-terminal variants may trigger kidney disease through interference with APOL3 control of actomyosin. Cell Rep. 30, 3821-36.e13 (2020)

122. Scales, S. J. et al. Apolipoprotein L1-specific antibodies detect endogenous APOL 1 inside the endoplasmic reticulum and on the plasma membrane of podocytes. J. Am. Soc. Nephrol. 31, 2044-2064 (2020).

123. Gupta, N. et al. Domain-specific antibodies reveal differences in the membrane topologies of apolipoprotein L1 in serum and podocytes. J. Am. Soc. Nephrol. 31, 2065-2082 (2020).

124. Sampson, M. G. et al. Integrative genomics identifies novel associations with APOL1 risk genotypes in black NEPTUNE subjects. J. Am. Soc. Nephrol. 27 , 814-823 (2016)

125. Ryu, J. H. et al. APOL1 renal risk variants promote cholesterol accumulation in tissues and cultured macrophages from APOL1 transgenic mice. PLoS One 14, e0211559 (2019).

126. Jha, A. et al. Alterations in plasma membrane ion channel structures stimulate NLRP3 inflammasomes activation in APOL1 risk milieu. FEBS J. 287, 2000-2022 (2020).

127. Lan, X. et al. Protein domains of APOL1 and its risk variants. Exp. Mol. Pathol. 99, 139-144 (2015).

128. Heneghan, J. F. et al. BH3 domain-independent apolipoprotein $\mathrm{L} 1$ toxicity rescued by $\mathrm{BCL} 2$ prosurvival proteins. Am. J. Physiol. Cell Physiol. 309 C332-C347 (2015)

129. Okamoto, K. et al. APOL1 risk allele RNA contributes to renal toxicity by activating protein kinase $\mathrm{R}$. Commun. Biol. 1, 188 (2018).

130. Zhang, J. Y. et al. UBD modifies $A P O L 1$-induced kidney disease risk. Proc. Natl Acad. Sci. USA 115 3446-3451 (2018).

131. Pollak, M. R., Genovese, G. \& Friedman, D. J. APOL1 and kidney disease. Curr. Opin. Nephrol. Hypertens. 21, 179-182 (2012)

132. Vanhollebeke, B. et al. Human Trypanosoma evansi infection linked to a lack of apolipoprotein L-I. N. Engl. J. Med. 355, 2752-2756 (2006).

133. Aghajan, M. et al. Antisense oligonucleotide treatment ameliorates IFN- $\gamma$-induced proteinuria in APOL1-transgenic mice. JCI Insight 4, e 126124 (2019).

134. US National Library of Medicine. ClinicalTrials.gov https://clinicaltrials.gov/ct2/show/NCT04269031 (2021)

135. US National Library of Medicine. ClinicalTrials.gov https://clinicaltrials.gov/ct2/show/NCT04340362 (2022).

136. Beckerman, P. \& Susztak, K. APOL1: the balance imposed by infection, selection, and kidney disease. Trends Mol. Med. 24, 682-695 (2018).

137. Friedman, D. J. \& Pollak, M. R. APOL1 and kidney disease: from genetics to biology. Annu. Rev. Physiol. 82, 323-342 (2020)

138. Nichols, B. et al. Innate immunity pathways regulate the nephropathy gene apolipoprotein L1. Kidney Int. 87, 332-342 (2015)

139. Davis, S. E., Khatua, A. K. \& Popik, W. Nucleosomal dsDNA stimulates APOL1 expression in human cultured podocytes by activating the cGAS/IFI16STING signaling pathway. Sci. Rep. 9, 15485 (2019).

140. Mathian, A., Hie, M., Cohen-Aubart, F. \& Amoura, Z. Targeting interferons in systemic lupus erythematosus: current and future prospects. Drugs 75, 835-846 (2015).

141. Rovin, B. H. \& Parikh, S. V. Lupus nephritis: the evolving role of novel therapeutics. Am. J. Kidney Dis. 63, 677-690 (2014)

142. Cui, D., Huang, G., Yang, D., Huang, B. \& An, B. Efficacy and safety of interferon-gamma-targeted therapy in Crohn's disease: a systematic review and meta-analysis of randomized controlled trials. Clin. Res. Hepatol. Gastroenterol. 37, 507-513 (2013).

143. Savva, A. \& Roger, T. Targeting toll-like receptors: promising therapeutic strategies for the management of sepsis-associated pathology and infectious diseases. Front. Immunol. 4, 387 (2013).

144. Norman, P. Selective JAK inhibitors in development for rheumatoid arthritis. Expert. Opin. Investig. Drugs 23, 1067-1077 (2014)

145. Moll, M. \& Kuemmerle-Deschner, J. B. Inflammasome and cytokine blocking strategies in autoinflammatory disorders. Clin. Immunol. 147, 242-275 (2013).

146. Olabisi, O. A. \& Heneghan, J. F. APOL1 nephrotoxicity: what does ion transport have to do with it? Semin. Nephrol. 37, 546-551 (2017).

147. Li, G. R. \& Dong, M. Q. Pharmacology of cardiac potassium channels. Adv. Pharmacol. 59, 93-134 (2010).

148. Kong, T. T., Zhang, C. M. \& Liu, Z. P. Recent developments of p38a MAP kinase inhibitors as antiinflammatory agents based on the imidazole scaffolds. Curr. Med. Chem. 20, 1997-2016 (2013).

149. Wen, $\mathrm{H}$. et al. APOL1 risk variants cause podocytes injury through enhancing endoplasmic reticulum stress. Biosci. Rep. 38, BSR20171713 (2018).

150. Zhuang, A. \& Forbes, J. M. Stress in the kidney is the road to pERdition: is endoplasmic reticulum stress a pathogenic mediator of diabetic nephropathy? J. Endocrinol. 222, R97-R111 (2014).

151. Scharff, D. P. et al. More than Tuskegee understanding mistrust about research participation. J. Health Care Poor Underserved 21, 879-897 (2010).

152. Umeukeje, E. M. et al. You are just now telling us about this? African American perspectives of testing for genetic susceptibility to kidney disease. J. Am. Soc Nephrol. 30, 526-530 (2019).

153. Young, B. A. et al. Apolipoprotein L1 testing in African Americans: involving the community in policy discussions. Am. J. Nephrol. 50, 303-311 (2019). 


\section{REVIEWS}

\section{Acknowledgements}

This work was supported by the Intramural Research Programs, NIDDK, NIH, Bethesda, MD, USA (ZO1 DK043308, to J.B.K.) and has been funded in whole or in part with federal funds from the National Cancer Institute, National Institutes of Health, under contract 75N91019D00024 (to C.A.W.). The content of this publication does not necessarily reflect the views or policies of the Department of Health and Human Services, nor does mention of trade names, commercial products or organizations imply endorsement by the
US Government. The authors also thank Al Kane, Scientific Publications, Graphics, and Media, Frederick Nationa Laboratory for Cancer Research and Erina He, Visual Arts, $\mathrm{NIH}$, for assistance with the figures before submission.

Author contributions

All authors contributed equally to this work.

Competing interests

The authors declare no competing interests.
Peer review information

Nature Reviews Nephrology thanks Opeyemi Olabisi, who co-reviewed with Somenath Datta, and the other, anonymous, reviewer(s) for their contribution to the peer review of this work.

Publisher's note

Springer Nature remains neutral with regard to jurisdictional claims in published maps and institutional affiliations.

(C) Springer Nature Limited 2022 